\title{
Interleukin-6 as an enhancer of anti-angiogenic therapy for ovarian clear cell carcinoma
}

\author{
Toshiyuki Seki $i^{1,4}$, Nozomu Yanaihara ${ }^{1,4 凶}$, Jason Solomon Shapiro, ${ }^{1,2}$, Misato Saito ${ }^{1}$, \\ Junya Tabata ${ }^{1}$, Ryo Yokomizo ${ }^{1}$, Daito Noguchi ${ }^{1}$, Takafumi Kuroda ${ }^{1}$, Ayako Kawabata ${ }^{1}$, \\ Jiro Suzuki ${ }^{1}$, Kazuaki Takahashi ${ }^{1}$, Haruka Matsuzawa ${ }^{3}$, Misayo Miyake ${ }^{3}$, Masataka Takenaka ${ }^{1}$, \\ Yasushi lida ${ }^{1}$, Satoshi Yanagida ${ }^{1}$ \& Aikou Okamoto ${ }^{1}$
}

Ovarian clear cell carcinoma (OCCC) is a subtype of epithelial ovarian cancer (EOC) that is associated with elevated interleukin-6 (IL-6) expression, resistance to chemotherapy, and increased mortality. Although bevacizumab (Bev) is a widely used anti-angiogenic agent for EOC, the efficacy of Bev and the role of IL- 6 in modulating angiogenesis in OCCC are unknown. We performed tube formation assays using human umbilical vein endothelial cells (HUVEC) cultured in OCCC cell-conditioned medium and using cells directly co-cultured with OCCC cells. We observed that IL-6 inhibition significantly mitigated the ability of Bev to impede tube formation in both cases. Furthermore, IL-6 blockade disrupted the anti-angiogenic efficacy of Bev and its concomitant anti-tumor activity. In addition, IL-6 inhibition resulted in a significant increase in angiopoietin-1 (Ang1) secretion and decreased vascular endothelial growth factor (VEGF) expression. Clinical specimens also exhibited this reciprocal relationship between IL- 6 and Ang1 expression. Finally, depletion of Ang1 abrogated the effects of IL- 6 inhibition on Bev activity, demonstrating that IL- 6 supports the anti-angiogenic activity of Bev by suppressing Ang1 expression and promoting dependence on VEGF for angiogenesis. Altogether, our data suggest that OCCC tumors with high IL-6 levels are candidates for Bev therapy.

Among the five distinct histotypes of epithelial ovarian cancer (EOC), ovarian clear cell carcinoma (OCCC) exhibits unique biological and molecular features, and is therefore recognized as a distinct entity presenting unique challenges for treatment ${ }^{1}$. The prevalence of OCCC varies geographically; while accounting for only $1-12 \%$ of EOC cases in Western countries, OCCC is relatively frequent among Asian EOC patients ${ }^{2}$. Until recently, few clinical trials have examined OCCC specifically and there is insufficient evidence regarding optimal chemotherapeutic approach, including molecular medicine. In general, OCCC is less sensitive to platinum-based first-line chemotherapy than other EOC histotypes and is associated with poor prognosis in advanced cases ${ }^{3}$. Identification of novel molecular targets related to carcinogenesis may help in patient stratification, prognosis, and treatment decisions. The AT-rich interactive domain $1 \mathrm{~A}$ gene (ARID1A) and the phosphatidylinositol 4,5-bisphosphate 3-kinase catalytic subunit a gene (PIK3CA) are frequently mutated in OCCC, and co-mutation in transgenic mice promotes OCCC tumor initiation, possibly by continuous stimulation of interleukin-6 (IL6)-dependent pro-inflammatory and pro-tumorigenic signaling as IL-6 is elevated in OCCC patients ${ }^{4}$. Moreover, the potential involvement of elevated IL-6/signal transducer and activator of transcription 3 (STAT3) pathway activity in OCCC pathogenesis has been reported by several groups ${ }^{5,6}$ and so may be a promising therapeutic target.

The anti-angiogenic drug bevacizumab (Bev), a monoclonal antibody against human vascular endothelial growth factor (VEGF), has been incorporated into first-line chemotherapy and follow-up maintenance therapy for advanced EOC. Although a randomized phase III trial evaluating the clinical benefits of Bev for EOC treatment (GOG-0218) did not show improvement in overall survival ${ }^{7}$, a recent retrospective plasma sample biomarker analysis from GOG-0218 patients found that high serum IL-6 concentration was predicted to have better anti-angiogenic treatment efficacy ${ }^{8}$. Thus, serum IL-6 may help to identify patients most responsive to

${ }^{1}$ Department of Obstetrics and Gynecology, The Jikei University School of Medicine, 3-25-8 Nishi-Shinbashi, Minato-ku, Tokyo 105-8461, Japan. ²Feinberg Cardiovascular Research Institute, Northwestern University, Chicago, IL 60611, USA. ${ }^{3}$ Department of Pathology, The Jikei University School of Medicine, Tokyo, Japan. ${ }^{4}$ These authors contributed equally: Toshiyuki Seki and Nozomu Yanaihara. ${ }^{\circledR}$ email: yanazou@jikei.ac.jp 
A

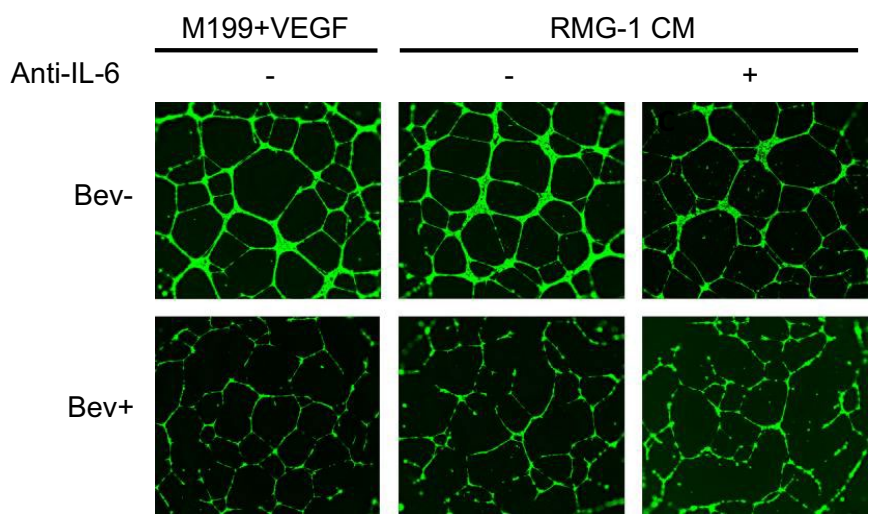

B

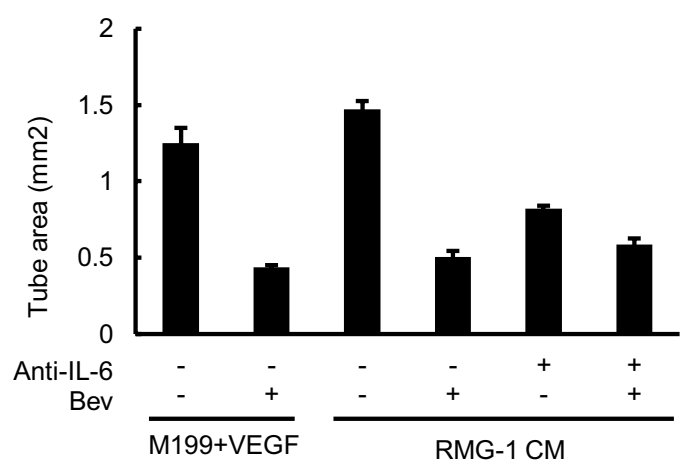

C

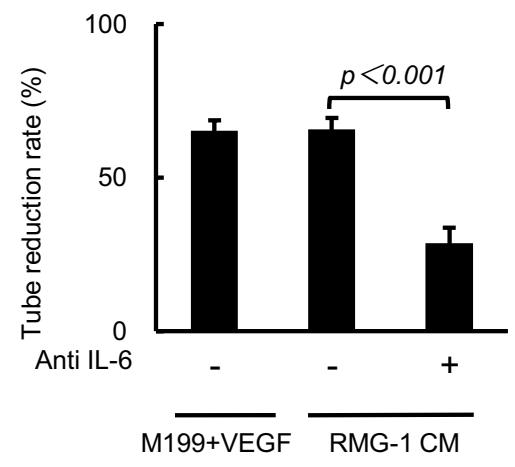

Figure 1. Attenuation of the anti-angiogenic function of Bev in culture media of IL-6 blocked OCCC cell. (A) Representative images of tube formation of HUVEC with the indicated culture media and reagents (magnification $\times 100$ ). Fluorescent microscope observation was made after $18 \mathrm{~h}$ of incubation and Calcein AM staining. (B) Tube areas in each well with indicated condition were measured by hybrid cell count software. Data were average of triplicated well. (C) The tube reduction rate by Bev treatment with or without IL-6 blockade. IL-6 blockade weakened Bev function. Data are shown from one of two independent experiments with similar results. Error bars are SEs. All the images were observed by fluorescence microscope (BZ-X800, Keyence, Osaka, JAPAN). Then, these obtained images were analyzed by the BZ-H4C analytic application (Keyence) for hybrid cell count and the BZ-H4CM application (Keyence) for macro cell count.

anti-angiogenic treatment. Further, these findings suggest that IL-6 signaling may potentiate the anti-angiogenic actions of VEGF blockade by Bev.

In this study, we examined the modulatory actions of IL- 6 on Bev anti-angiogenic efficacy by conducting tube formation assays in co-cultures of OCCC cell lines and human umbilical vein endothelial cells (HUVEC) in the presence or absence of Bev and/or anti-IL-6 antibody. Results suggested that IL-6 promotes the anti-angiogenic efficacy of Bev by suppressing angiopoietin-1 (Ang1) release. Further, we present evidence that IL-6 also suppresses Ang1 production in human OCCC tissue. Collectively, these results provide valuable information for the development of individualized OCCC treatment strategies.

\section{Results}

Attenuation of Bev anti-angiogenic efficacy by IL- 6 signal blockade. To investigate the influence of IL-6 signaling on anti-angiogenic therapy in OCCC, we first assayed the level of IL-6 production of a series of OCCC cell lines. Among 5 cell lines, we chose RMG-1 cell with intermediate level of IL-6 production for further experiments (Supplementary Fig. S1A). Then, we examined the capacity of HUVECs to form tubular networks in the presence of conditioned medium from the OCCC cell line RMG-1 (Fig. 1A). Briefly, RMG-1 cells were cultured in $1 \%$ oxygen with or without anti-IL- 6 antibody and the supernatant (conditioned medium) was used for tube formation assays. The ability of HUVEC cells to form tubular networks in RMG-1 conditioned media without IL-6 signal inhibition was equivalent to that in recombinant VEGF-supplemented culture medium as indicated by tube area measurements (Fig. 1A,B). As expected, the addition of Bev strongly impeded tube formation both in VEGF-supplemented medium and RMG-1 conditioned medium without IL-6 signal inhibition, suggesting that RMG-1 cells may also secrete VEGF (Fig. 1B). However, the efficacy of Bev to impede tube formation was strongly mitigated by RMG-1 condition medium with anti-IL- 6 antibody, from $66 \pm 3.8 \%$ in the 
A

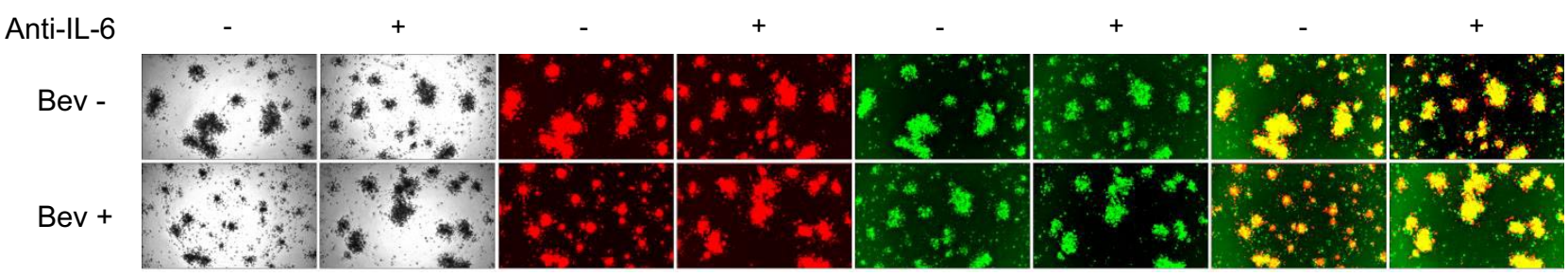

B

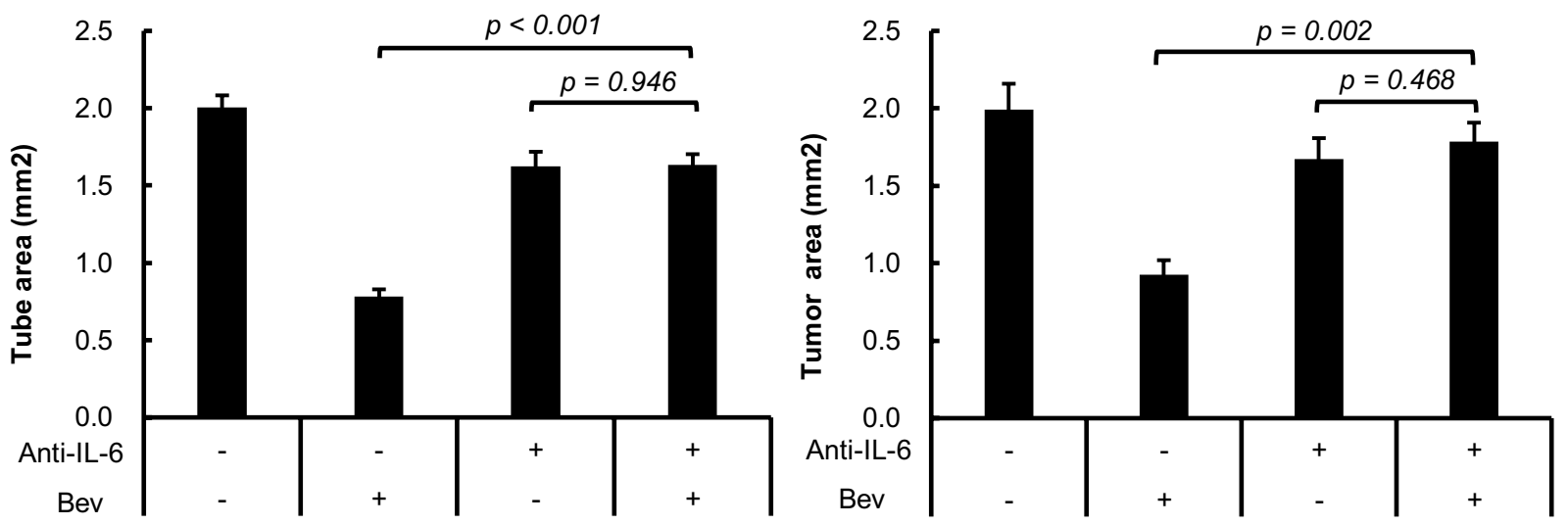

Figure 2. The role of IL-6 signal for the anti-angiogenic and the anti-tumor effects of Bev in $3 \mathrm{D}$ co-culture system. (A) Representative micrograph of 3D co-culture with or without IL-6 blockade and/or Bev observed through light microscope and fluorescent microscope at experimental day 4. HUVEC was stained by Dil, emitting red fluorescence. RMG-1/GFP was seen as green fluorescence. Overlaid images of Dil and GFP were shown at the rightmost. (B) Measured area at day 4 of each color by hybrid cell count software. Dil-stained area, regarded as the tube area, is shown on the left and GFP area, regarded as the tumor area, is shown on the right side. Error bars are SEs. All the images were observed by fluorescence microscope (BZ-X800, Keyence). Then, these obtained images were analyzed by the BZ-H4C analytic application (Keyence) for hybrid cell count and the BZ-H4CM application (Keyence) for macro cell count.

absence of IL- 6 signal blockade to only $29 \% \pm 5.0 \%$ in the presence of anti-IL-6 $(p<0.001)$ (Fig. 1C). Moreover, this mitigation by anti-IL-6 antibody was dose dependent (Supplementary Fig. S2A,B).

Attenuation of Bev anti-angiogenic and anti-tumor efficacy under IL- 6 signal blockade. Findings from Fig. 1 suggest that IL- 6 signaling potentiates the anti-angiogenic efficacy of Bev. To clarify the underlying mechanisms as well as the potential effects of IL-6 signaling on Bev anti-tumor activity, we examined these drugs in a co-culture system of the OCCC cell line RMG-1/GFP (emitting green fluorescence) and HUVEC prestained with Dil (emitting red fluorescence) (Fig. 2A). In this system, the tumor cell line attached to HUVEC and demonstrated proliferation, implying a direct interaction between the two (Supplementary Fig. S3A). Consistent with the results shown in Fig. 1, Bev treatment alone for 4 days reduced the tube area and this anti-angiogenic effect was significantly mitigated by anti-IL-6 $(p<0.001)$. Thus, reversal of Bev-mediated suppression of angiogenesis by IL- 6 signal blockade was maintained in the presence of tumor cells. Moreover, the tumor area was also significantly larger in the combined Bev and anti-IL-6-treated group than in the groups treated with Bev alone $(p=0.002)$ (Fig. 2B), suggesting that IL-6 blockade disrupted both the anti-angiogenic and concomitant antitumor effects of Bev. Both effects of IL-6 signal blockade on Bev activity were observed over the entire 7-days experimental period (Supplementary Fig. S3B,C).

IL-6 signaling blockade enhanced Ang1 release from tumor cells. To further investigate the mechanisms underlying IL-6-dependent suppression of Bev anti-angiogenic activity, we examined the effects of IL-6 on secretion of the angiogenic modulators VEGF, Ang1/2, and osteopontin by tumor and HUVEC. Production of VEGF by RMG-1 cells was only mildly suppressed by anti-IL-6 antibody treatment as evidenced by ELISA analysis of culture supernatant (Fig. 3A), whereas osteopontin production by RMG-1 tumor cells was significantly suppressed by anti-IL-6 $(p<0.01)$ (Fig. 3B). In contrast, anti-IL-6 antibody treatment enhanced Ang1 production $(p<0.05)$ (Fig. 3C). HUVECs did not produce detectable VEGF or osteopontin, while Ang1 production was increased and Ang-2 production decreased by anti-IL-6 antibody treatment (Fig. 3A-D). These results suggest that the anti-angiogenic activity of Bev is mitigated by IL-6 signal blockade through enhanced produc- 

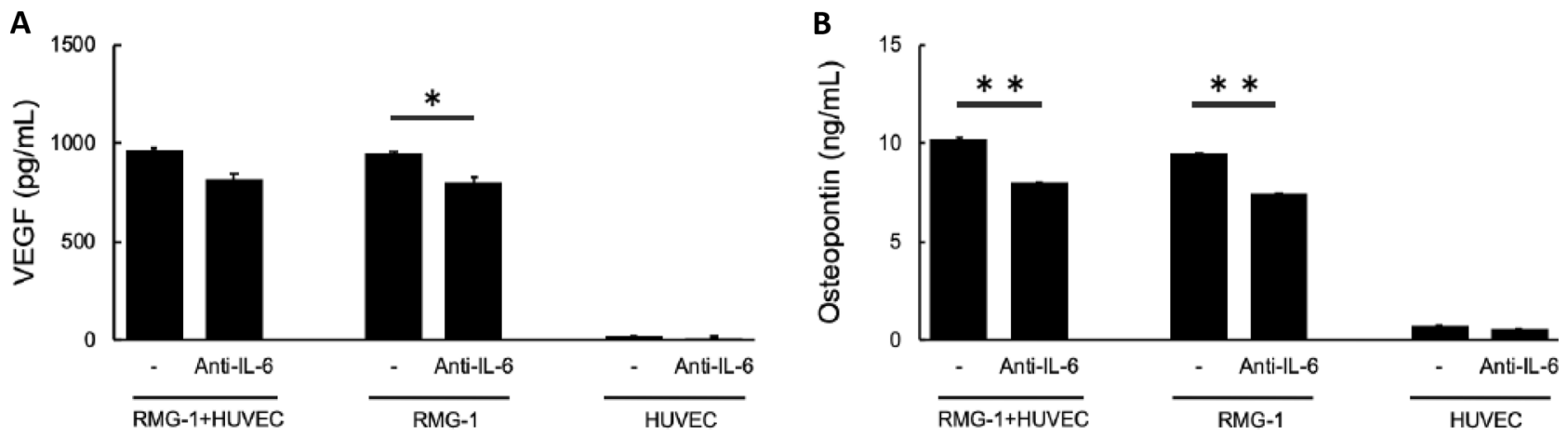

C
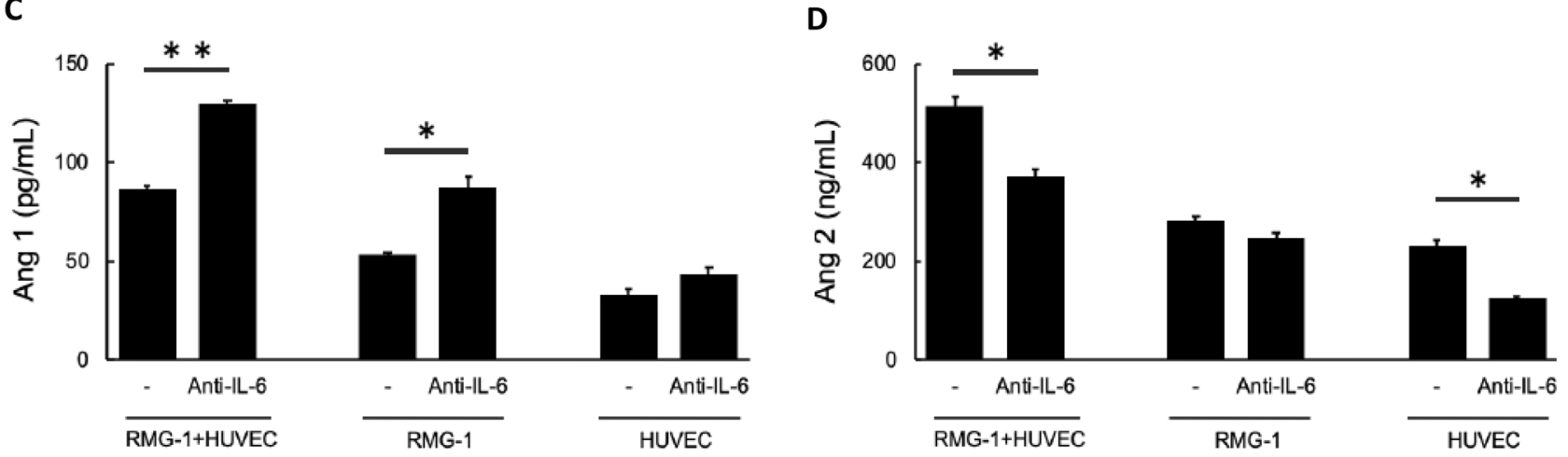

Figure 3. Angiogenic factors released by RMG-1 and HUVEC. (A) VEGF, (B) osteopontin, (C) Ang1, and (D) Ang2 production from RMG-1 and HUVEC in response to IL-6 signal blockade determined by ELISA assay. Data of HUVEC was calculated by the subtraction the data of RMG-1 mono-culture from that of RMG-1 + HUVEC. Error bars are SEs. ${ }^{\star} \mathrm{P}<0.05,{ }^{\star *} \mathrm{P}<0.005$.

tion of Ang1 (rather than VEGF production). To further assess IL- 6 effects on Ang1 production by OCCC cells, these ELISA assays were repeated on several other OCCC cell lines. In general, these assays indicated that IL-6 and Ang1 production are reciprocally regulated. While OVTOKO and HAC-2 lines produced little IL-6, both produced high levels of Ang1. Conversely, the OVISE line produced a large amount of IL- 6 and a small amount of Ang1 (Supplementary Fig. S1A). Only RMG-1 and -2 cells produced moderate amounts of both IL-6 and Ang1. Moreover, anti-IL-6 antibody treatment enhanced Ang1 production in RMG-1, RMG-2, and OVISE cells (Supplementary Fig. S1B).

We then examined the relationship between IL- 6 and Ang1 expression in 60 OCCC clinical tissue samples. Of the 13 tumor samples with high IL-6 expression, only one sample exhibited high Ang1 expression as evidenced by immunohistochemical staining. Further, Ang1 expression was significantly lower among tumor samples with high IL-6 expression than in samples with low IL-6 expression $(p=0.021)$ (Supplementary Fig. S4A,B). This result suggested that Bev may be more efficacious in tumors with high IL-6 expression because of the suppressive effect of IL-6 on pro-angiogenic Ang1 release.

Suppression of Ang1 release by targeted siRNA restored the anti-angiogenic effects of Bev. To confirm that IL-6 potentiates the anti-angiogenic efficacy of Bev by suppressing Ang1 release, we conducted tube formation assays after manipulating Ang1 expression using targeted and control siRNAs. First, we confirmed the feasibility of this strategy by demonstrating that the targeted siRNA (siAng1) suppresses the production of Ang1 from RMG-1 with or without IL-6 signaling blockade (Supplementary Fig. S5). In the presence of conditioned medium from RMG-1 cells transfected with siAng1 and treated with anti-IL-6 antibody, Bev reduced the tube area by $55 \%$, similar to the findings in assays using conditioned medium from tumor cells that were not treated with anti-IL-6 (Fig. 4A-C). In other words, suppression of Ang1 accumulation in the conditioned medium restored the anti-angiogenic efficacy of Bev in the presence of anti-IL-6, providing further evidence that IL-6 indirectly promotes the anti-angiogenic activity of Bev by suppressing Ang 1 secretion by OCCC cells.

\section{Discussion}

Molecular profiling of OCCC tumor tissues has revealed several potential prognostic biomarkers, predictors of treatment response, and therapeutic targets ${ }^{9-14}$. For instance, studies have reported the hyperactivation of several signaling pathways, including hypoxia-inducible factor $1 \alpha$ (HIF-1a)/VEGF and IL-6/STAT3 pathways in OCCC ${ }^{15-17}$. Mabuchi et al. reported that OCCC cells under intratumoral hypoxia strongly expressed VEGF and that Bev demonstrated anti-tumor efficacy against OCCC both in vitro and in vivo ${ }^{18}$. However, they also suggested that 
A

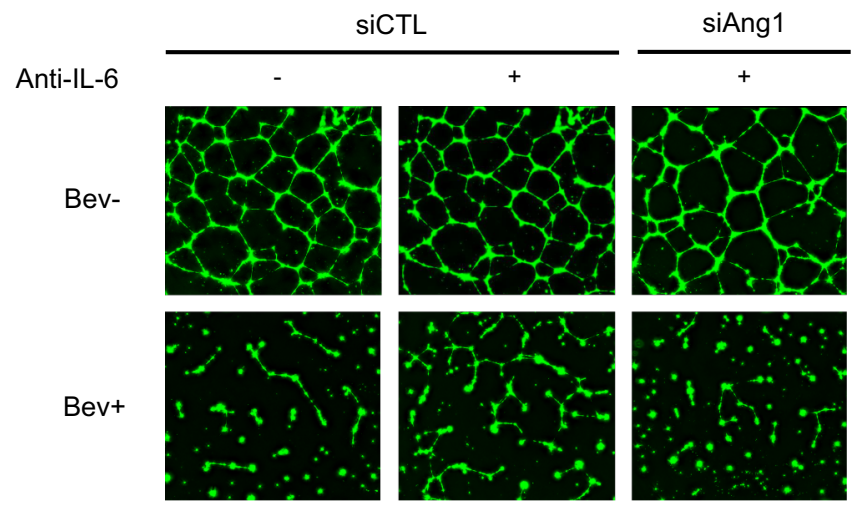

B

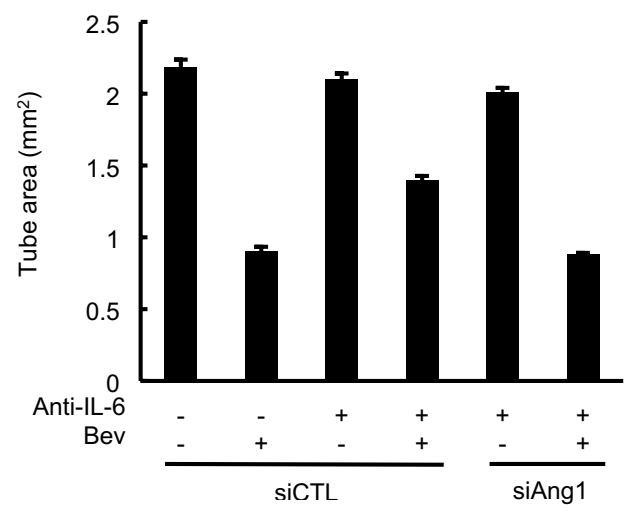

C

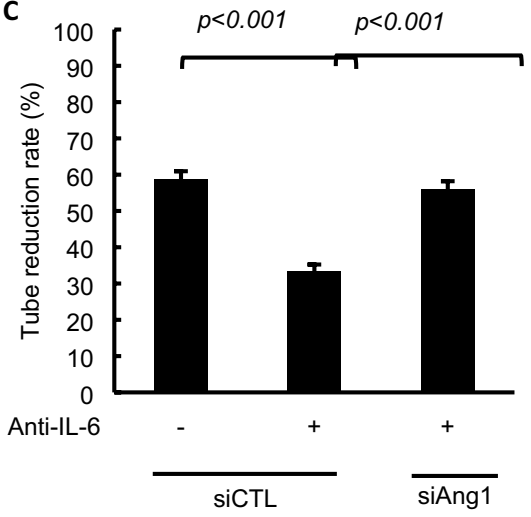

Figure 4. The role of Ang1 in IL-6 mediated Bev anti-angiogenic effect enhancement. (A) Images of tubes formed by HUVEC in the conditioned media from RMG- 1 treated by indicated siRNA and anti-IL- 6 antibody (magnification $\times 40$ ). Fluorescent microscope observation was made after $18 \mathrm{~h}$ of incubation and Calcein AM staining. (B) Tubes in each well with indicated condition were measured by hybrid cell count software in order to elucidate the tube area. Data were average of triplicated well. (C) Reduction rate of tube area by Bev treatment with or without IL-6 blockade and siRNA, IL-6 blockade weakened Bev function in siCTL whereas siAng1 restored the anti-angiogenic function. Data are shown from one of two independent experiments with similar results. siCTL: siRNA without gene silencing ability (control). siAng1: angiopoietin-1 silencing siRNA. Error bars are SEs. All the images were observed by fluorescence microscope (BZ-X800, Keyence, Osaka, JAPAN). Then, these obtained images were analyzed by the BZ-H4C analytic application (Keyence) for hybrid cell count and the BZ-H4CM application (Keyence) for macro cell count.

VEGF may not be a reliable biomarker for predicting Bev sensitivity. Therefore, although anti-angiogenic treatment by Bev appears promising based on pathogenesis, there is still no widely recognized biomarker predictive of its clinical efficacy. Here we demonstrate that high IL-6 or low Ang1 may be such predictors.

Upregulation of IL- 6 and related pathway mediators as well as the anti-tumor efficacy of IL-6 pathway inhibition have been reported in OCCC $5,6,19,20$. In addition, several studies have documented significant associations between poor OCCC prognosis and high IL-6 in tumor or serum samples ${ }^{5,11,20}$. Anglesio et al. reported upregulation of the IL-6/STAT3/HIF pathway and therapeutic responses to the anti-angiogenic agent sunitinib in two chemotherapy-resistant OCCC cases $^{5}$. However, the phase II GOG-254 trial evaluating this multi-receptor tyrosine kinase inhibitor for the treatment of persistent or recurrent OCCC found minimal clinical efficacy ${ }^{21}$. Recently, a retrospective biomarker analysis revealed longer survival by Bev-treated patients with high plasma IL-6 level compared to those with lower plasma IL- $6^{8}$. Based on these findings, we evaluated the influence of IL- 6 on anti-angiogenic and anti-tumor efficacies of Bev using an in vitro OCCC model to reveal potential molecular mechanisms, and further examined the association of IL- 6 expression with that of the pro-angiogenic factor Angl among OCCC patients to help identify good candidates for Bev treatment.

In general, IL-6 has been demonstrated to influence angiogenesis as a pro-angiogenic factor ${ }^{22}$. For instance, transgenic mice engineered to overexpress IL- 6 exhibited hypervascularization of the cerebellum ${ }^{23}$, whereas IL-6-deficient mice exhibited reduced angiogenic responses to wound injury ${ }^{24}$. Further, IL- 6 induced VEGF production by tumor cells and consequently activated angiogenesis ${ }^{25,26}$. In the current study, IL- 6 signaling blockade reduced the anti-angiogenic activity and associated anti-tumor activity of Bev in vitro co-culture system of OCCC cells and HUVECs. However, the moderate alteration of VEGF production by OCCC cells under IL-6 blockade suggested that some other angiogenic factor(s) were responsible for the observed attenuation of the anti-angiogenic activity of Bev. Several VEGF-independent angiogenic mechanisms of IL- 6 have been reported, 

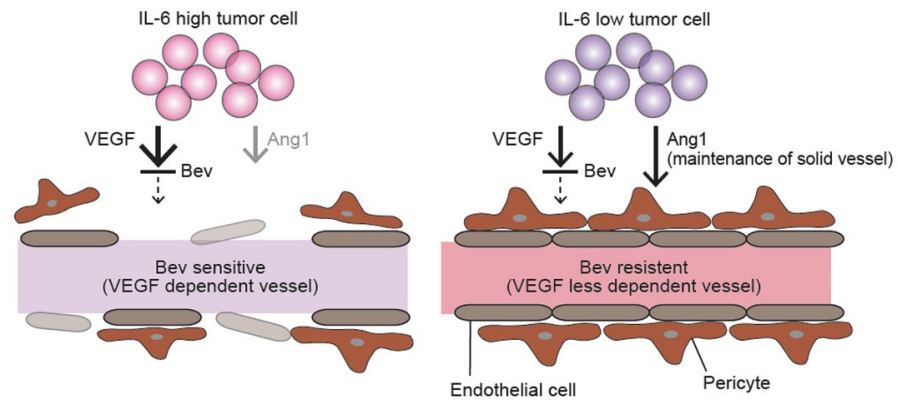

Figure 5. Mechanism of enhanced anti-angiogenic efficacy of Bev in IL-6 high tumor with Ang1 suppression. IL-6 supports the anti-angiogenic activity of Bev by suppressing Ang1 expression and promoting dependence on VEGF for angiogenesis. (This illustration is drawn by means of Adobe Illustrator).

including Ang1 modulation, and Kayakabe et al. reported that IL-6 could destabilize angiogenesis by inhibiting Ang1 signaling in a co-culture model of rheumatoid arthritis ${ }^{27}$. Additional analyses in our model suggested that IL-6 promoted the anti-angiogenic activity of Bev by suppressing Ang1 release from OCCC cells. Furthermore, the association of high IL-6 expression with low Ang1 expression was found in a series of specimens from patients with OCCC. According to these results, Angl may interfere with the function of Bev in OCCC (Fig. 5).

Ang1 is believed to facilitate vessel stabilization, and its signaling is believed to support the induction and growth of tumor vasculature even under VEGF blockade, resulting in improved tumor perfusion ${ }^{28}$. Huang et al. also reported that Ang1 protected the tumor vasculature from regression, increased vessel caliber, and induced the recruitment of mural cells under anti-VEGF treatment ${ }^{29}$. Moreover, Casanovas et al. found that tumors exhibiting continued progression during anti-VEGF receptor-2 antibody treatment maintained substantial Ang1 expression $^{30}$. According to these findings, Ang1 suppression may be important for Bev to exert its effects. In the current study, we found that IL-6 blockade enhanced Ang1 production by tumor cells, whereas siRNA-induced Ang1 depletion restored the impaired anti-angiogenic function of Bev under IL- 6 blockade. Collectively, IL-6 signaling could reduce the pro-angiogenic function of Ang1 in tumors and relatively enhance that of VEGF, which in turn enhances Bev function in OCCC cells.

The utility of IL-6 as a potential predictor of anti-angiogenic drug response is controversial. Earlier reports suggested that high IL-6 levels could be predictive of a survival benefit of Bev treatment in patients with EOC ${ }^{8}$, metastatic renal cancer ${ }^{31}$, and metastatic colorectal cancer ${ }^{10}$. Conversely, other studies reported that low IL-6 levels were associated with better Bev treatment responses in hepatocellular carcinoma ${ }^{32}$, pancreatic cancer ${ }^{33}$, and metastatic colorectal cancer ${ }^{34}$. In addition, a recent clinical study found that aflibercept, a recombinant fusion protein that blocks the VEGF pathway in advanced EOC, was more effective in patients with low IL-6 levels ${ }^{35}$. Differences in cellular IL-6 responses among tumor types, study design, or clinical stage may explain these discordant results. Further research is warranted to elucidate the mechanism by which IL-6 modulates Ang1 and the prognostic utility of IL- 6 as a biomarker of anti-angiogenic drug response.

In conclusion, this study demonstrated that IL- 6 enhanced the anti-angiogenic efficacy of Bev by suppressing Angl in addition to increasing VEGF production in OCCC cells. Furthermore, the current study provides a strong rationale for prospective clinical trials of anti-angiogenic therapy for EOC, including OCCC, to clarify the prognostic utility of IL- 6 and Ang1 levels.

\section{Materials and methods}

Cell culture. The human OCCC cell lines RMG-1 and RMG-2 were kindly provided by Dr. D. Aoki (Keio University, Tokyo, Japan) and maintained in Ham's F12 (GIBCO BRL, Grand Island, NY, USA) supplemented with $10 \%$ fetal bovine serum (FBS) (Cytiva, Marlborough, MA, USA). The OVTOKO and OVISE lines were purchased from the Japanese Collection of Research Bioresources Cell Bank (Osaka, Japan), and the HAC-2 line was kindly provided by Dr. M. Nishida (Tsukuba University, Tsukuba, Japan). These three cell lines were maintained in RPMI-1640 medium (Sigma-Aldrich, St Louis, MO, USA) supplemented with 10\% FBS. For experiments, all OCCC cell lines were pre-incubated for $24 \mathrm{~h}$ in M199 medium (Sigma-Aldrich) supplemented with $0.5 \%$ bovine serum albumin (BSA) (Sigma-Aldrich) under a hypoxic environment $\left(1 \% \mathrm{O}_{2}\right)$ created by the BIONIX hypoxic culture kit (SUGIYAMA-GEN, Tokyo, Japan). To modulate IL-6 signaling, the indicated concentration of anti-IL-6 antibody (MAB206-100, clone 6708; R\&D Systems, Minneapolis, MN) was added to the medium and culture continued for $48 \mathrm{~h}$. Other cultures were incubated for the same period but without antiIL-6 treatment. Subsequently, supernatants were collected as conditioned media (with or without anti-IL-6) and stored frozen for further experiments. An RMG-1 line expressing green fluorescent protein (RMG-1/GFP) was purchased from Anticancer Japan (Chiba, Japan) and treated in the same manner as RMG-1 cells. HUVEC cells were kindly provided by Dr. I. Yamamoto (The Jikei University School of Medicine, Tokyo, Japan) and maintained in endothelial cell medium (Sciencell Research Laboratory, Carlsbad, CA) with 5\% FBS.

Transfection and siRNA. A predesigned small interfering RNA (siRNA) targeting angiopoietin-1 (siAng1) (Silencer Select Pre-Designed siRNA, Assay ID: s1356) and a non-targeted control siRNA (siCTL) (Silencer 
Select Negative Control, \#4390843) were purchased from ThermoFisher Scientific (Waltham, MA). Prior to transfection, siRNAs were premixed with lipofectamine RNAiMAX (ThermoFisher Scientific) plus Opti-MEM (ThermoFisher Scientific). RMG-1 cells were then transfected by incubation with this mixture for $24 \mathrm{~h}$ under a hypoxic environment. After an additional $24 \mathrm{~h}$ of incubation, the culture medium was exchanged for fresh medium with or without $100 \mathrm{ng} / \mathrm{mL}$ anti-IL-6 antibody as indicated. The supernatants from these cultures were collected as conditioned media for tube formation assays.

Antibodies and angiogenic factor. Recombinant human VEGF (aa207-318) was purchased from PeproTech (Rocky Hill, NJ). The human VEGF-A monoclonal antibody Bev was kindly provided by Chugai Pharmaceutical Co., Ltd (Tokyo, Japan).

Tube formation assay. Tube formation assays were conducted as described by Yin et al. ${ }^{36}$. Briefly, Corning 96-well flat bottom plates (Corning, NY) were coated with $30 \mu \mathrm{L}$ growth factor-reduced Matrigel matrix (Corning) and seeded with $1 \times 10^{5}$ HUVEC in $100 \mu \mathrm{L}$ RMG-1 conditioned medium supplemented with $0.5 \%$ BSA. Recombinant VEGF $(5 \mathrm{ng} / \mathrm{mL})$ and Bev $(10 \mu \mathrm{g} / \mathrm{mL})$ were added as indicated for specific experiments. After $18 \mathrm{~h}$ of incubation, tubes were stained with Calcein AM (Corning) to quantify total tube area using a fluorescence microscope (BZ-X800, Keyence, Osaka, JAPAN) and the BZ-H4C analytic application (https://www.keyence. com/products/microscope/fluorescence-microscope/bz-x700/models/bz-h4c/) for hybrid cell count and the BZ-H4CM application (https://www.keyence.com/products/microscope/fluorescence-microscope/bz-x700/ models/bz-h4cm/) for macro cell count.

Enzyme-linked immunosorbent assays (ELISAs). ELISAs were performed to measure the medium concentrations of angiogenic factors secreted by RMG-1, RMG-2, OVISE, OVTOKO, and HAC-2 cell lines. Briefly, cells were seeded in T-25 flasks at $5 \times 10^{5}$ cells per flask. After $24 \mathrm{~h}$ of incubation, the culture medium was exchanged for M199 medium plus 0.5\% BSA with or without anti-IL-6 antibody $(100 \mathrm{ng} / \mathrm{mL})$ as indicated and incubation was continued under hypoxia for an additional $48 \mathrm{~h}$. Part of the culture supernatant was then collected for ELISA analysis of VEGF, IL-6, Ang1, angiopoietin-2 (Ang2), and osteopontin concentrations using specific ELISA kits (DVE00 for VEGF, D6050 for IL-6, DANG10 for Ang1, DANG20 for Ang2, and DOST00 for osteopontin; all from R\&D Systems). The remaining supernatants were collected as conditioned media for HUVEC culture. HUVEC were initially seeded at $1 \times 10^{5}$ cells per T- 25 flask. After $24 \mathrm{~h}$ of incubation, the culture medium was exchanged for RMG-1 cell-conditioned medium and incubation continued for an additional $48 \mathrm{~h}$. The supernatants were then collected for analysis using the same ELISA kits.

Co-culture assay. A 3D-culture protocol was performed based on the Matrigel sandwich structure method described previously ${ }^{37}$. Briefly, $30 \mu \mathrm{L}$ Matrigel was added into each well of a 96-well plate pre-chilled on ice. The plates were then incubated at $37^{\circ} \mathrm{C}$ for $30 \mathrm{~min}$ to enable polymerization of this Matrigel basal layer. HUVEC cells were pre-stained with Corning DilC12(3) Fluorescent Dye (Dil), suspended at $2 \times 10^{4}$ cells per $75 \mu \mathrm{L}$ M199 medium supplemented with $1 \%$ FBS, and seeded onto the basal layer. After $4 \mathrm{~h}$ of incubation to allow tube formation, the same number of RMG-1/GFP cells suspended in $75 \mu$ l pre-chilled M199 medium containing $1 \%$ FBS, $10 \mu \mathrm{g} / \mathrm{mL} \mathrm{Bev}$, and $10 \%$ Matrigel with or without $100 \mathrm{ng} / \mathrm{mL}$ anti-IL- 6 antibody were seeded onto the polymerized Matrigel base layer. Then, the plates were incubated at $37^{\circ} \mathrm{C}$ to allow polymerization of the top layer. The co-culture system was maintained in M199 medium containing $1 \% \mathrm{FBS}$ at $37^{\circ} \mathrm{C}$ under a $5 \% \mathrm{CO}_{2}$ atmosphere for 7 days with medium exchange every other day. The Dil-stained area and GFP-stained area, corresponding to tube area and tumor area, respectively, were quantified using a fluorescence microscope (BZ-X800, Keyence) and the BZ-H4C and BZ-H4CM analytic applications.

Clinical samples and immunohistochemistry. Analysis of human tumor samples was approved by the ethics committee of The Jikei University School of Medicine (32-017(10092)). Sixty stage II-IV OCCC patients receiving primary surgical resection followed by post-operative adjuvant chemotherapy at The Jikei University School of Medicine and affiliated hospitals from 2013 to 2018 were enrolled. Paraffin-embedded tumor samples from primary surgery were stained with hematoxylin-eosin to confirm the diagnosis of OCCC. To examine IL- 6 and Angl expression, formalin-fixed, paraffin-embedded tissue sections ( $4 \mu \mathrm{m}$ thick) were deparaffinized, incubated in Cell Conditioning 1 (CC1) standard solution (citrate buffer $\mathrm{pH}$ 8.5, Ventana Medical Systems) for $60 \mathrm{~min}$ at $100{ }^{\circ} \mathrm{C}$ for antigen retrieval, and then incubated in antibodies against IL-6 (1:400, 21865-1-AP; Proteintech) and Ang1 (1:200, ab8451; Abcam, Cambridge, UK). Immunostained slides were evaluated by two independent pathologists (H.M and M.M) blinded to clinical information. Staining scores were standardized by comparison between the observers, and discrepancies were resolved by reevaluating the slides using a multihead microscope. Immunoreactivity for IL-6 in the tumor cytoplasm was categorized as none/focal (0\%-40\%) or diffuse $(50 \%-100 \%)^{11}$. Immunostaining for Ang1 was assessed in a semi-quantitative manner as previously described $^{38}$. Tumor and stromal areas were evaluated independently and each section was assigned two scores: Staining intensity ( 0 , no staining; 1 , weak staining; 2 , moderate staining; 3 , intense staining) and proportion of cells stained ( 0 , no cells staining; $1,1 \%-25 \% ; 2,26 \%-50 \% ; 3,51 \%-75 \% ; 4,76 \%-100 \%)$. The scores for staining intensity and proportion of stained cells were multiplied to yield individual tumor and stromal area staining scores for Ang1. The two scores were then added to yield a final Ang1 staining score for each sample, which was then categorized as high $(>8)$ or low $(0-7)$. 
Statistical analysis. All data are expressed as the mean \pm standard error of the mean. Means of experimental and control groups were compared by independent samples Student's t-test, one-way analysis of variance followed by post hoc Bonferroni's multiple comparison tests, or Fischer's exact tests as indicated. A p $<0.05$ (two-tailed) was considered statistically significant for all tests. All statistical analyses were performed using EZR software (Saitama Medical Centre, Jichi Medical University; http://www.jichi.ac.jp/saitama-sct/SaitamaHP. files/ statmedOSX.html, Kanda, 2012), a graphical interface for R (The R foundation for Statistical Computing. Vienna, Austria, ver. 3.2.2).

Received: 3 December 2020; Accepted: 16 March 2021

Published online: 08 April 2021

\section{References}

1. del Carmen, M. G., Birrer, M. \& Schorge, J. O. Clear cell carcinoma of the ovary: A review of the literature. Gynecol. Oncol. 126, 481-490 (2012).

2. Okamoto, A. et al. Gynecologic cancer intergroup (GCIG) consensus review for clear cell carcinoma of the ovary. Int. J. Gynecol. Cancer 24, S20-S25 (2014).

3. Winter, W. E. et al. Prognostic factors for stage III epithelial ovarian cancer: A gynecologic oncology group study. J. Clin. Oncol. 25, 3621-3627 (2007).

4. Chandler, R. L. et al. Coexistent ARID1A-PIK3CA mutations promote ovarian clear-cell tumorigenesis through pro-tumorigenic inflammatory cytokine signalling. Nat. Commun. 6, 6118 (2015).

5. Anglesio, M. S. et al. IL6-STAT3-HIF signaling and therapeutic response to the angiogenesis inhibitor sunitinib in ovarian clear cell cancer. Clin. Cancer Res. 17, 2538-2548 (2011).

6. Yanaihara, N. et al. Cytokine gene expression signature in ovarian clear cell carcinoma. Int. J. Oncol. 41, 1094-1100 (2012).

7. Burger, R. A., Fleming, G. F., Mannel, R. S., Greer, B. E. \& Liang, S. X. Incorporation of bevacizumab in the primary treatment of ovarian cancer. N. Engl. J. Med. 11, 1 (2011).

8. Alvarez Secord, A. et al. Predictive blood-based biomarkers in patients with epithelial ovarian cancer treated with carboplatin and paclitaxel with or without bevacizumab: results from GOG-0218. Clin. Cancer Res. https://doi.org/10.1158/1078-0432.CCR-19$0226(2020)$.

9. Ayhan, A. et al. CCNE1 copy-number gain and overexpression identify ovarian clear cell carcinoma with a poor prognosis. Mod. Pathol. 30, 297-303 (2017)

10. Liu, Y. et al. Correlation of angiogenic biomarker signatures with clinical outcomes in metastatic colorectal cancer patients receiving capecitabine, oxaliplatin, and bevacizumab. Cancer Med. 2, 234-242 (2013).

11. Kawabata, A. et al. Prognostic impact of interleukin-6 expression in stage I ovarian clear cell carcinoma. Gynecol. Oncol. 146, 609-614 (2017).

12. Ogiwara, H. et al. Targeting the vulnerability of glutathione metabolism in ARID1A-deficient cancers. Cancer Cell 35, 177-190.e8 (2019).

13. Williamson, C. T. et al. ATR inhibitors as a synthetic lethal therapy for tumours deficient in ARID1A. Nat. Commun. 7, 13837 (2016).

14. Bitler, B. G. et al. ARID1A-mutated ovarian cancers depend on HDAC6 activity. Nat. Cell Biol. 19, 962-973 (2017).

15. Kuroda, T. \& Kohno, T. Precision medicine for ovarian clear cell carcinoma based on gene alterations. Int. J. Clin. Oncol. 25, 419-424 (2020).

16. Takahashi, K., Takenaka, M., Kawabata, A., Yanaihara, N. \& Okamoto, A. Rethinking of treatment strategies and clinical management in ovarian clear cell carcinoma. Int. J. Clin. Oncol. 25, 425-431 (2020).

17. Gershenson, D. M., Okamoto, A. \& Ray-Coquard, I. Management of rare ovarian cancer histologies. J. Clin. Oncol. 37, 2406-2415 (2019).

18. Mabuchi, S. et al. vascular endothelial growth factor is a promising therapeutic target for the treatment of clear cell carcinoma of the ovary. Mol. Cancer Ther. 9, 2411-2422 (2010).

19. Yamaguchi, K. et al. Identification of an ovarian clear cell carcinoma gene signature that reflects inherent disease biology and the carcinogenic processes. Oncogene 29, 1741-1752 (2010).

20. Yanaihara, N. et al. Antitumor effects of interleukin-6 (IL-6)/interleukin-6 receptor (IL-6R) signaling pathway inhibition in clear cell carcinoma of the ovary. Mol. Carcinog. 55, 832-841 (2016).

21. Chan, J. K. et al. A phase II evaluation of sunitinib in the treatment of persistent or recurrent clear cell ovarian carcinoma: An NRG Oncology/Gynecologic Oncology Group Study (GOG-254). Gynecol. Oncol. 150, 247-252 (2018).

22. Middleton, K., Jones, J., Lwin, Z. \& Coward, J. I. G. Interleukin-6: An angiogenic target in solid tumours. Crit. Rev. Oncol. Hematol. 89, 129-139 (2014).

23. Campbell, I. L. et al. Neurologic disease induced in transgenic mice by cerebral overexpression of interleukin 6. Proc. Natl. Acad. Sci. 90, 10061-10065 (1993).

24. Lin, Z.-Q., Kondo, T., Ishida, Y., Takayasu, T. \& Mukaida, N. Essential involvement of IL-6 in the skin wound-healing process as evidenced by delayed wound healing in IL-6-deficient mice. J. Leukoc. Biol. 73, 713-721 (2003).

25. Cohen, T., Nahari, D., Cerem, L. W., Neufeld, G. \& Levi, B.-Z. Interleukin 6 induces the expression of vascular endothelial growth factor. J. Biol. Chem. 271, 736-741 (1996).

26. Xu, Q. et al. Targeting Stat3 blocks both HIF-1 and VEGF expression induced by multiple oncogenic growth signaling pathways. Oncogene 24, 5552-5560 (2005).

27. Kayakabe, K. et al. Interleukin- 6 promotes destabilized angiogenesis by modulating angiopoietin expression in rheumatoid arthritis. Rheumatology 51, 1571-1579 (2012).

28. Winkler, F. et al. Kinetics of vascular normalization by VEGFR2 blockade governs brain tumor response to radiation. Role of oxygenation, angiopoietin-1, and matrix metalloproteinases. Cancer Cell 6, 553-563 (2004).

29. Huang, J. Angiopoietin-1/Tie-2 activation contributes to vascular survival and tumor growth during VEGF blockade. Int. J. Oncol. https://doi.org/10.3892/ijo_00000131 (1992).

30. Casanovas, O., Hicklin, D. J., Bergers, G. \& Hanahan, D. Drug resistance by evasion of antiangiogenic targeting of VEGF signaling in late-stage pancreatic islet tumors. Cancer Cell 8, 299-309 (2005).

31. Nixon, A. B. et al. Identification of predictive biomarkers of overall survival (OS) in patients (pts) with advanced renal cell carcinoma (RCC) treated with interferon alpha (I) with or without bevacizumab (B): Results from CALGB 90206 (Alliance). J. Clin. Oncol. 31, 15 (2013) (Suppl; Abstr 4520).

32. Boige, V. et al. Efficacy, safety, and biomarkers of single-agent bevacizumab therapy in patients with advanced hepatocellular carcinoma. Oncologist 17, 1063-1072 (2012). 
33. Nixon, A. B. et al. Prognostic and predictive blood-based biomarkers in patients with advanced pancreatic cancer: Results from CALGB80303 (alliance). Clin. Cancer Res. 19, 6957-6966 (2013).

34. Abajo, A. et al. Identification of predictive circulating biomarkers of bevacizumab-containing regimen efficacy in pre-treated metastatic colorectal cancer patients. Br. J. Cancer 107, 287-290 (2012).

35. Eichten, A. et al. Resistance to anti-VEGF therapy mediated by autocrine IL6/STAT3 signaling and overcome by IL6 blockade. Cancer Res. 76, 2327-2339 (2016).

36. Yin, L. et al. Fasudil inhibits vascular endothelial growth factor-induced angiogenesis in vitro and in vivo. Mol. Cancer Ther. 6, $1517-1525$ (2007).

37. Lee, G. Y., Kenny, P. A., Lee, E. H. \& Bissell, M. J. Three-dimensional culture models of normal and malignant breast epithelial cells. Nat. Methods 4, 359-365 (2007).

38. Magkouta, S. et al. Targeting Tie-2/angiopoietin axis in experimental mesothelioma confers differential responses and raises predictive implications. Oncotarget 9, 21783-21796 (2018).

\section{Acknowledgments}

We thank all members of the Obstetrics and Gynecology Department at The Jikei University School of Medicine for their enthusiastic clinical practice.

\section{Author contributions}

Conception and design: N.Y, T.S, and J.S.S. Acquisition of data: T.S, M.S, D.N, H.M, M.M, A.K, and M.T. Analysis and interpretation of data: N.Y, M.S, and T.S. Writing, review, and/or revision of the manuscript: T.S, N.Y, J.T, R.Y, T.K, J.S, K.T, Y.I, S.Y, J.S.S, and A.O.

\section{Funding}

This study was supported by Japan Society for the Promotion of Science KAKENHI Grant Number 20K09610 (NY) and by The Jikei University Research Fund (NY).

\section{Competing interests}

The authors declare no competing interests.

\section{Additional information}

Supplementary Information The online version contains supplementary material available at https://doi.org/ 10.1038/s41598-021-86913-9.

Correspondence and requests for materials should be addressed to N.Y.

Reprints and permissions information is available at www.nature.com/reprints.

Publisher's note Springer Nature remains neutral with regard to jurisdictional claims in published maps and institutional affiliations.

(c) (i) Open Access This article is licensed under a Creative Commons Attribution 4.0 International

License, which permits use, sharing, adaptation, distribution and reproduction in any medium or format, as long as you give appropriate credit to the original author(s) and the source, provide a link to the Creative Commons licence, and indicate if changes were made. The images or other third party material in this article are included in the article's Creative Commons licence, unless indicated otherwise in a credit line to the material. If material is not included in the article's Creative Commons licence and your intended use is not permitted by statutory regulation or exceeds the permitted use, you will need to obtain permission directly from the copyright holder. To view a copy of this licence, visit http://creativecommons.org/licenses/by/4.0/.

(c) The Author(s) 2021 